Article

\title{
Polymorphisms in ERCC5 rs17655 and ERCC1 rs735482 Genes Associated with the Survival of Male Patients with Postoperative Oral Squamous Cell Carcinoma Treated with Adjuvant Concurrent Chemoradiotherapy
}

\author{
Thomas Senghore ${ }^{1,2}{ }^{\mathbb{D}}$, Huei-Tzu Chien ${ }^{3,4}$, Wen-Chang Wang ${ }^{5}$, You-Xin Chen ${ }^{1}$, \\ Chi-Kuang Young ${ }^{6}$, Shiang-Fu Huang ${ }^{3,7, *}$ and Chih-Ching Yeh ${ }^{1,8, *(D)}$ \\ 1 School of Public Health, College of Public Health, Taipei Medical University, Taipei 11031, Taiwan; \\ tsenghore@gmail.com (T.S.); youxin810@gmail.com (Y.-X.C.) \\ 2 Department of Nursing, School of Medicine and Allied Health Sciences, University of The Gambia, \\ Independence Drive, Banjul, P.O. Box 1646, The Gambia \\ 3 Department of Public Health, Chang Gung University, Tao-Yuan 33305, Taiwan; kathy.htchien@gmail.com \\ 4 Department of Nutrition and Health Sciences, Chang Gung University of Science and Technology, \\ Taoyuan 33302, Taiwan \\ 5 Ph.D. Program for Translational Medicine, College of Medical Science and Technology, \\ Taipei Medical University, Taipei 11031, Taiwan; wangwc@tmu.edu.tw \\ 6 Department of Otolaryngology, Chang Gung Memorial Hospital, Keelung 20401, Taiwan; \\ rioriorioman@gmail.com \\ 7 Department of Otolaryngology, Head and Neck Surgery, Chang Gung Memorial Hospital, Linkou, \\ Taoyuan 33305, Taiwan \\ 8 Department of Public Health, College of Public Health, China Medical University, Taichung 40402, Taiwan \\ * Correspondence: shiangfu.huang@gmail.com (S.-F.H.); ccyeh@tmu.edu.tw (C.-C.Y.); \\ Tel.: +886-3-3281200 (S.-F.H.); +886-2-7361661 (ext. 6534) (C.-C.Y.)
}

Received: 26 November 2018; Accepted: 25 December 2018; Published: 1 January 2019

\begin{abstract}
The nucleotide excision repair (NER) pathway plays a major role in the repair of DNA damaged by exogenous agents, such as chemotherapeutic and radiotherapeutic agents. Thus, we investigated the association between key potentially functional single nucleotide polymorphisms (SNPs) in the NER pathway and clinical outcomes in oral squamous cell carcinoma (OSCC) patients treated with concurrent chemoradiotherapy (CCRT). Thirteen SNPs in five key NER genes were genotyped in 319 male OSCC patients using iPLEX MassARRAY. Cox proportional hazards models and Kaplan-Meier survival curves were used to estimate the risk of death or recurrence. Carriers of the XPC rs2228000 TT genotype showed a borderline significant increased risk of poor overall survival under the recessive model (hazard ratio $(\mathrm{HR})=1.81,95 \%$ confidence interval $(\mathrm{CI})=0.99-3.29$ ). The CC genotypes of ERCC5 rs17655 ( $\mathrm{HR}=1.54,95 \% \mathrm{CI}=1.03-2.29)$ and $E R C C 1 \mathrm{rs735482}(\mathrm{HR}=1.65$, $95 \% \mathrm{CI}=1.06-2.58)$ were associated with an increased risk of worse disease-free survival under the recessive model. In addition, participants carrying both the CC genotypes of ERCC5 rs17655 and $E R C C 1 \mathrm{rs735482}$ exhibited an enhanced susceptibility for recurrence $(\mathrm{HR}=2.60,95 \% \mathrm{CI}=1.11-6.09)$. However, no statistically significant interaction was observed between them. Our findings reveal that the ERCC5 rs17655 CC and ERCC1 rs735482 CC genotypes were associated with an increased risk of recurrence in male patients with OSCC treated with CCRT. Therefore, CCRT may not be beneficial, and alternative treatments are required for such patients.
\end{abstract}

Keywords: nucleotide excision repair; genetic polymorphism; oral squamous cell carcinoma; concurrent chemoradiotherapy; prognosis 


\section{Introduction}

Oral squamous cell carcinoma (OSCC) is the leading cause of cancer morbidity and mortality, especially among men in Taiwan [1]. Despite new advances in the diagnosis and therapeutic approaches, the 5-year survival remains low [1,2]. While relapse of OSCC remains a major clinical challenge, the incidence of relapse among patients varies, even for those with a similar stage of disease at diagnosis or those who undergo the same treatment [3]. This implies that other factors, such as genetic variations, may play an important role in disease prognosis.

Most patients with OSCC are diagnosed at an advanced stage of the disease [4]. For these patients, the treatment options are limited to mainly systemic therapy, often as concurrent chemoradiotherapy (CCRT) with platinum-based DNA damaging agents as either primary treatment or adjuvant postoperative therapy [5-7]. However, the overall survival (OS) for these patients remains poor because most of them experience recurrence or distance metastases [8-10]. Genetic variations in DNA repair genes affect susceptibility to the efficacy and survival outcome of a certain treatment [11,12]. Increased DNA repair capacity may affect the sensitivity of the tumor cells to chemotherapy and radiotherapy (RT) by allowing cancer cells to repair DNA that has been damaged by these agents. Single nucleotide polymorphism (SNP) in genes involved in the nucleotide excision repair (NER) pathway may modulate DNA repair capacity by influencing gene expression or activity, thereby affecting the anticancer effects of therapeutic agents and treatment response $[13,14]$.

The excision repair cross-complementation genes, including groups 1 (ERCC1), 2 (ERCC2), and 5 (ERCC5) and xeroderma pigmentosium complementation group $\mathrm{A}(X P A)$ and $\mathrm{C}(\mathrm{XPC})$ encode proteins that are involved in the NER pathway; and together with other proteins, operate to recognize and repair damaged DNA [15]. The XPC together with XPF initially recognize the DNA lesion that is unwound and remodeled by helicase proteins ERCC 3 and ERCC2 that binds to XPA and replication protein A (RPA). The ERCC1 and ERCC5 proteins are involved in the incision of the identified DNA lesion. The difference in treatment response and clinical outcome have been attributed to SNPs in genes that code of the above proteins $[13,14]$. Therefore, identifying genetic markers in the NER pathway may help develop personalized management strategies, thereby maximizing treatment success and improving survival.

Thus, we conducted a retrospective cohort study to test whether SNPs in genes involved in the NER pathway are associated with prognosis in male patients with OSCC treated with adjuvant CCRT. A total of 13 SNPs in ERCC5, ERCC2, ERCC1, XPC, and XPA genes, which have been found to affect the risk and/or survival of cancers, were selected in the present study $[13,14,16-20]$. Their associations with clinical outcomes were evaluated using alternative genetic models, including additive, dominant, and recessive models.

\section{Material and Methods}

\subsection{Study Population}

In total, 360 male participants newly diagnosed with histopathological confirmed advanced OSCC who received surgery plus adjuvant CCRT were recruited from the Head and Neck Surgery Department's Cancer Registry at Chang Gung Memorial Hospital, LinKou, Taiwan, from 1999 to 2016. A total of 41 participants were excluded, including 13 of aboriginal ethnicity, 23 with early-stage oral cancer (TNM stages I and II), and 5 with missing information on clinicopathologic variables (TNM stage, vascular invasion, and extracapsular spread). A final sample of 319 was included for analysis. Information on demographic characteristics (age, education, occupation, and ethnicity), lifestyle habit (cigarette smoking, alcohol drinking, and betel nut chewing), and family cancer history were collected through an interviewer-administered questionnaire. Lifestyle habits were categorized as either never (if the person never engaged in the habit continuously for more than a year) or ever (if the person ever engaged in the habit for more than a year). From the weight and height measurements, body mass index (BMI) was calculated as weight $/$ height ${ }^{2}\left(\mathrm{~kg} / \mathrm{m}^{2}\right)$. Clinical information was also 
collected before treatment through a detailed medical history, physical examination, completed blood count, routine blood chemistry, computed tomography (CT) or magnetic resonance imaging (MRI) of the head and neck, abdominal ultrasound, and whole body bone scan or positron emission tomography scan. This study was approved by the Chang Gung Memorial Hospital (IRB No. 201800213B0) and Taipei Medical University ethics review committees (IRB No. N201802083). All participants provided written informed consent after a detailed explanation of study objectives.

\subsection{Sample Preparation and DNA Extraction}

For each participant, a pair of tumor and normal adjacent nontumor tissue samples were surgically removed, dissected into small pieces, and immediately stored in liquid nitrogen at $-80^{\circ} \mathrm{C}$. The surgically removed samples were then sent for pathological examination and staging as per the seventh edition of the American Joint Committee on Cancer-TNM staging system [21]. Histology diagnosis was defined as squamous cell carcinoma, verrucous carcinoma, cylindric cell carcinoma, adenoid cystic carcinoma, mucoepidermoid carcinoma, and adenocarcinoma. For this study, only those with a diagnosis of squamous cell carcinoma were included. Venous blood samples were also collected and stored in heparinized tubes. Germline DNA was extracted from buffer-coated cells using the standard phenol-chloroform method and prepared for genotyping.

\subsection{SNP Selection and Genotyping}

SNPs in the NER pathway were selected from studies that indicated that SNPs were associated with the risk or prognosis of malignancies in ethnic Chinese [16,18-20]. A total of 13 potentially functional SNPs in ERCC5 (rs2094258, rs1047768, rs17655, and rs873601), ERCC2 (rs13181 and rs1799793), ERCC1 (rs735482, rs3212986, and rs11615), XPC (rs2228001 and rs2228000), and XPA (rs1800975 and rs10817938) genes were genotyped using the Sequenom iPLEX MassARRAY system (Sequenom, Inc., San Diego, CA, USA). A 10\% random sample was reanalyzed, and it showed 100\% concordance for all the polymorphisms.

\subsection{Patient Treatment and Follow-Up}

All patients underwent radical tumor excision with clinical stage-based neck dissection after preoperative tumor survey. The primary tumors were resected above 1-cm safety margins (both peripheral and deep margins). Neck dissections were performed according to examination status. If the lesion invaded deeply and crossed the midline, as observed in tongue cancer, bilateral neck dissection was performed. Pathologic parameters included tumor stage, nodal status, extranodal extension (ENE), tumor cell differentiation, perineural invasion, skin invasion, bone invasion, and tumor depth. Postoperative RT was administered to patients with pT4 stage tumor, pathologically close margins $(\leq 4 \mathrm{~mm})$, or pathologically positive lymph nodes. The radiation dose lay between 6000 and 6600 cGy. CCRT with cisplatin-based agents was administered to patients with ENE or pathological multiple lymph node metastases 4 to 8 weeks after the surgical procedure. During the course of RT, 5-fluorouracil was administered orally.

Following commencement of treatment, the participants were monitored during their treatment and after treatment through regular clinical and radiological examinations. Follow-up involved monthly checkups for the first 6 months. This was followed by checkups every 2 months in the second 6 months, then checkups every 3 months within the second year, and checkups every 6 months thereafter. The follow-up included an analysis of medical history, physical examination (including complete oral examination), laboratory examination, X-rays, and CT or MRI. To confirm recurrence, histology of biopsy or imaging studies were conducted. Data for all deaths resulting from OSCC were based on death certificates. 


\subsection{Statistical Analysis}

Statistical analysis was performed using SAS (version 9.4 for Windows; SAS institute, Cary, NC, USA). Demographic and clinical characteristics were summarized as mean and standard deviation for continuous variables and frequency and proportions for categorical variables. The distribution of genotypes by clinical characteristics was assessed using Chi-square test. Major clinical outcomes were disease-free survival (DFS) and OS. DFS was measured from the first day of treatment to the time of recurrence, metastasis, or death due to any cause. OS was calculated as the time elapsed (in months) from the date of commencing RT to the date of death. Patients without an event at the date of the last contact were considered as being censured or subject to administrative censoring by the end of the follow-up period. Survival analysis was conducted using the Kaplan-Meier method, and survival curve differences among the genotypes were compared using the log-rank test. Univariate and multivariate Cox proportional hazards models were used to evaluate the effects of demographic, clinical characteristics, and SNPs on survival. Hazard ratios (HRs) and their 95\% confidence intervals (CIs) were used to estimate the relative risk of death or recurrence. We evaluated the individual variants in different genetic models, including additive, dominant, and recessive models, on OSCC survival. Sociodemographic and clinical factors significant in the univariate analysis were adjusted in multivariate Cox regression models. Furthermore, multiplicative interactions were evaluated using the likelihood ratio test. Due to the location of multiple SNPs within the same chromosome or gene, linkage disequilibrium (LD) analysis was performed using Haploview (version 4.2). For those SNPs within the same block that were found to be in high LD with each other, further haplotypes analysis was performed using PHASE software (version 2.1) [22]. Statistical significance was set at $p<0.05$ and was two-sided.

\section{Results}

\subsection{Demographic and Clinical Characteristics of Study Participants}

The demographic and clinical characteristics of the study patients are summarized in Table 1. The mean age was $49.72 \pm 9.8$. Most participants were under 50 years $(51.41 \%)$ old, of Taiwanese descent (paternal $72.10 \%$ and maternal $74.92 \%$ ), had normal BMI $(49.22 \%)$, had smoked cigarettes (according to the "ever" criterion; 85.25\%), drank alcohol (69.28\%), and chewed betel nut (86.21\%). A considerable number of patients exhibited poor clinical characteristics. In total, 55 (17.24\%) had poorly differentiated tumors, 197 (61.76\%) had primary tumor size in the T3 to T4 range, $217(67.92 \%)$ had N2 to N3 nodal involvement, 177 (55.49\%) had perineural invasion, 19 (5.96\%) had vascular invasion, 40 (12.54\%) had lymphatic invasion, 205 (64.24\%) had ENE, and 277 (86.83\%) had pathologic TNM stage IV. The genotype frequency distribution analysis showed a statistically significant difference in genotypes of ERCC1 rs11615 in terms of tumor differentiation $(p=0.039), X P C$ rs2228000 in terms of vascular invasion $(p=0.045), E R C C 1 \mathrm{rs} 3212986$ and XPA rs10817938 in terms of lymphatic invasion $(p=0.046$ and 0.033 , respectively), XPC rs2228001 in terms of pathologic TNM stage $(p=0.039)$, and ERCC5 rs17655 in terms of DFS ( $p=0.049)$ (Table S1).

\subsection{Survival Analysis}

The median (range) follow-up duration was 15 months (1-199 months) and 12 months (1-199 months) for OS and DFS, respectively. In the univariate analysis, N2-N3 nodal involvement $(\mathrm{HR}=2.41,95 \% \mathrm{CI}=1.42-4.10)$, lymphatic invasion $(\mathrm{HR}=2.18,95 \% \mathrm{CI}=1.30-3.67)$, and $\mathrm{ENE}$ $(\mathrm{HR}=3.91,95 \% \mathrm{CI}=2.13-7.19)$ were significantly associated with $\mathrm{OS}$, whereas primary tumor size in the range of $\mathrm{T} 3$ to $\mathrm{T} 4(\mathrm{HR}=1.72,95 \% \mathrm{CI}=1.17-2.53), \mathrm{N} 2-\mathrm{N} 3$ nodal involvement $(\mathrm{HR}=1.63$, $95 \% \mathrm{CI}=1.09-2.44)$, and ENE ( $\mathrm{HR}=1.79,95 \% \mathrm{CI}=1.20-2.69)$ were significantly associated with DFS. However, no significant association was observed between demographic and lifestyle factors and survival (Table 2). 
Table 1. Demographic and clinical characteristics of patients with oral squamous cell carcinoma (OSCC) treated with concurrent chemoradiotherapy (CCRT).

\begin{tabular}{|c|c|}
\hline Variable & $n(\%)$ \\
\hline Total & $319(100)$ \\
\hline Mean age (SD), years & $49.72(9.8)$ \\
\hline \multicolumn{2}{|l|}{ Age, years } \\
\hline$<50$ & $164(51.41)$ \\
\hline$\geq 50$ & 155 (48.59) \\
\hline \multicolumn{2}{|l|}{ Ethnicity of father } \\
\hline Taiwanese & $230(72.10)$ \\
\hline Hakka & $72(22.57)$ \\
\hline Mainland Chinese & $17(5.33)$ \\
\hline \multicolumn{2}{|l|}{ Ethnicity of mother } \\
\hline Taiwanese & $239(74.92)$ \\
\hline Hakka & $74(23.20)$ \\
\hline Mainland Chinese & $6(1.88)$ \\
\hline \multicolumn{2}{|l|}{ BMI, $\mathrm{kg} / \mathrm{m}^{2}$} \\
\hline$<18.5$ & $22(6.90)$ \\
\hline 18.5-23.9 & $157(49.22)$ \\
\hline$\geq 24$ & $140(43.89)$ \\
\hline \multicolumn{2}{|l|}{ Cigarette smoking } \\
\hline Never & $47(14.73)$ \\
\hline Ever & $272(85.27)$ \\
\hline \multicolumn{2}{|l|}{ Alcohol drinking } \\
\hline Never & $98(30.72)$ \\
\hline Ever & $221(69.28)$ \\
\hline \multicolumn{2}{|l|}{ Betel nut chewing } \\
\hline Never & $44(13.79)$ \\
\hline Ever & $275(86.21)$ \\
\hline \multicolumn{2}{|l|}{ Tea drinking } \\
\hline Never & $163(51.10)$ \\
\hline Ever & $156(48.90)$ \\
\hline \multicolumn{2}{|l|}{ Coffee drinking } \\
\hline Never & $243(76.18)$ \\
\hline Ever & $76(23.82)$ \\
\hline \multicolumn{2}{|l|}{ Tumor differentiation } \\
\hline Well differentiated & $51(15.99)$ \\
\hline Moderate & $210(65.83)$ \\
\hline Poor & $55(17.24)$ \\
\hline Unclear & $3(0.94)$ \\
\hline \multicolumn{2}{|l|}{ Primary tumor size } \\
\hline $\mathrm{T} 1-\mathrm{T} 2$ & $122(38.24)$ \\
\hline T3-T4 & $197(61.76)$ \\
\hline \multicolumn{2}{|l|}{ Nodal involvement } \\
\hline N0-N1 & 102 (31.97) \\
\hline N2-N3 & $217(67.92)$ \\
\hline \multicolumn{2}{|l|}{ Perineural invasion } \\
\hline No & $142(44.51)$ \\
\hline Yes & $177(55.49)$ \\
\hline \multicolumn{2}{|l|}{ Vascular invasion } \\
\hline No & $300(94.04)$ \\
\hline Yes & $19(5.96)$ \\
\hline \multicolumn{2}{|l|}{ Lymphatic invasion } \\
\hline No & $279(87.46)$ \\
\hline Yes & $40(12.54)$ \\
\hline \multicolumn{2}{|l|}{ Extranodal extension } \\
\hline No & $114(35.74)$ \\
\hline Yes & $205(64.26)$ \\
\hline \multicolumn{2}{|l|}{ Pathologic TNM stage } \\
\hline III & $42(13.17)$ \\
\hline IV & $277(86.83)$ \\
\hline
\end{tabular}

OSCC, oral squamous cell carcinoma; SD, standard deviation; BMI, body mass index. 
Table 2. Univariate association of demographic and clinical factors with survival in patients with OSCC treated with CCRT.

\begin{tabular}{|c|c|c|c|c|}
\hline \multirow{2}{*}{ Variable } & \multicolumn{2}{|c|}{ Overall Survival } & \multicolumn{2}{|c|}{ Disease-Free Survival } \\
\hline & HR $(95 \% \mathrm{CI})$ & $p$ Value & HR $(95 \% \mathrm{CI})$ & $p$ Value \\
\hline \multicolumn{5}{|l|}{ Age } \\
\hline$<50$ & 1.00 & & 1.00 & \\
\hline$\geq 50$ & $0.67(0.44-1.03)$ & 0.068 & $0.81(0.57-1.15)$ & 0.239 \\
\hline \multicolumn{5}{|l|}{ Ethnicity of father } \\
\hline Taiwanese & 1.00 & & 1.00 & \\
\hline Hakka & $0.88(0.51-1.50)$ & 0.633 & $0.66(0.40-1.08)$ & 0.101 \\
\hline Mainland Chinese & $1.27(0.55-2.93)$ & 0.577 & $1.72(0.94-3.14)$ & 0.079 \\
\hline \multicolumn{5}{|l|}{ Ethnicity of mother } \\
\hline Taiwanese & 1.00 & & 1.00 & \\
\hline Hakka & $0.75(0.43-1.29)$ & 0.295 & $0.67(0.42-1.08)$ & 0.100 \\
\hline Mainland Chinese & $1.80(0.57-5.72)$ & 0.320 & $1.60(0.59-4.37)$ & 0.355 \\
\hline \multicolumn{5}{|l|}{ BMI, kg $/ \mathrm{m}^{2}$} \\
\hline $18.5-23.9$ & 1.00 & & 1.00 & \\
\hline$<18.5$ & $0.85(0.36-1.98)$ & 0.705 & $1.49(0.79-2.84)$ & 0.223 \\
\hline$\geq 24$ & $0.66(0.42-1.03)$ & 0.068 & $0.84(0.58-1.23)$ & 0.372 \\
\hline \multicolumn{5}{|l|}{ Cigarette smoking } \\
\hline Never & 1.00 & & 1.00 & \\
\hline Ever & $0.89(0.51-1.57)$ & 0.675 & $1.02(0.62-1.68)$ & 0.938 \\
\hline \multicolumn{5}{|l|}{ Alcohol drinking } \\
\hline Never & 1.00 & & 1.00 & \\
\hline Ever & $0.99(0.64-1.54)$ & 0.971 & $1.10(0.75-1.61)$ & 0.636 \\
\hline \multicolumn{5}{|l|}{ Betel nut chewing } \\
\hline Never & 1.00 & & 1.00 & \\
\hline Ever & $1.09(0.58-2.05)$ & 0.794 & $1.40(0.80-2.45)$ & 0.240 \\
\hline \multicolumn{5}{|l|}{ Tea drinking } \\
\hline Never & 1.00 & & 1.00 & \\
\hline Ever & $0.93(0.61-1.41)$ & 0.727 & $1.07(0.75-1.53)$ & 0.701 \\
\hline \multicolumn{5}{|l|}{ Coffee drinking } \\
\hline Never & 1.00 & & 1.00 & \\
\hline Ever & $0.65(0.37-1.13)$ & 0.123 & $1.27(0.85-1.89)$ & 0.251 \\
\hline \multicolumn{5}{|l|}{ Tumor differentiation } \\
\hline Well differentiated & 1.00 & & 1.00 & \\
\hline Moderate & $0.94(0.55-1.60)$ & 0.819 & $0.89(0.56-1.44)$ & 0.645 \\
\hline Poor & $0.84(0.40-1.74)$ & 0.629 & $1.22(0.68-2.18)$ & 0.500 \\
\hline Unclear & $2.80(0.37-1.19)$ & 0.319 & $1.66(0.22-2.39)$ & 0.621 \\
\hline \multicolumn{5}{|l|}{ Primary tumor size } \\
\hline $\mathrm{T} 1-\mathrm{T} 2$ & 1.00 & & 1.00 & \\
\hline T3-T4 & $1.29(0.83-2.01)$ & 0.258 & $1.72(1.17-2.53)$ & $0.006 *$ \\
\hline \multicolumn{5}{|l|}{ Nodal involvement } \\
\hline N0-N1 & 1.00 & & 1.00 & \\
\hline N2-N3 & $2.41(1.42-4.10)$ & $0.001 *$ & $1.63(1.09-2.44)$ & 0.018 * \\
\hline \multicolumn{5}{|l|}{ Perineural invasion } \\
\hline No & 1.00 & & 1.00 & \\
\hline Yes & $1.29(0.85-1.98)$ & 0.238 & $1.27(0.89-1.83)$ & 0.189 \\
\hline \multicolumn{5}{|l|}{ Vascular invasion } \\
\hline No & 1.00 & & 1.00 & \\
\hline Yes & $1.43(0.66-3.10)$ & 0.366 & $0.43(0.43-2.00)$ & 0.856 \\
\hline \multicolumn{5}{|l|}{ Lymphatic invasion } \\
\hline No & 1.00 & & 1.00 & \\
\hline Yes & $2.18(1.30-3.67)$ & $0.003 *$ & $1.37(0.82-2.28)$ & 0.233 \\
\hline Extranodal extension & & & & \\
\hline No & 1.00 & & 1.00 & \\
\hline Yes & $3.91(2.13-7.19)$ & $<0.001 *$ & $1.79(1.20-2.69)$ & $0.005 *$ \\
\hline Pathologic TNM stage & & & & \\
\hline III & 1.00 & & 1.00 & \\
\hline IV & $1.64(0.81-3.29)$ & 0.168 & $1.66(0.93-2.96)$ & 0.087 \\
\hline
\end{tabular}

BMI, body mass index; OSCC, oral squamous cell carcinoma; HR, hazard ratio; CI, confidence interval. * $p<0.05$.

In the univariate Cox proportional hazards models, the ERCC1 rs735482 CC genotype was marginally significantly associated with poor $\mathrm{DFS}(\mathrm{HR}=1.53,95 \% \mathrm{CI}=0.99-2.38 ; p=0.058)$. The XPA rs10817938 CC genotype was significantly associated with an increased risk of worse OS (HR $=2.97$, $95 \% \mathrm{CI}=1.20-7.35 ; p=0.019)$, and DFS (HR $=2.61,95 \% \mathrm{CI}=1.06-6.41 ; p=0.037)$, respectively (Table S2). 
The results for the multivariate Cox proportional hazards models with covariates adjusted for all selected SPNs are shown in Table 3. Only the XPC rs2228000 TT genotype (HR $=1.81$, $95 \% \mathrm{CI}=0.99-3.29, p=0.053$ ) showed an increased risk of poor OS at borderline significance compared with the CC+CT genotypes. The ERCC5 rs17655 CC $(\mathrm{HR}=1.50,95 \% \mathrm{CI}=1.01-2.24 ; p=0.045)$ and ERCC1 rs735482 CC (HR $=1.61,95 \% \mathrm{CI}=1.04-2.51 ; p=0.034)$ genotypes were significantly associated with an increased risk of DFS compared with their counterparts with the GG+GC and $\mathrm{AA}+\mathrm{AC}$ genotypes, respectively, in the recessive models. The test of LD show that SNPs in ERCC5 block 1 (rs2094258 and rs1047768; $\mathrm{D}^{\prime}=0.97, R^{2}=0.19$ ) and block 2 (rs17655 and rs873601; $\mathrm{D}^{\prime}=0.98$, $\left.R^{2}=0.89\right), E R C C 1$ block (rs3212986 and rs11615; $\left.\mathrm{D}^{\prime}=1.00, R^{2}=0.18\right), X P C$ block (rs2228001 and rs2228000; $\left.\mathrm{D}^{\prime}=1.00, R^{2}=0.28\right)$, and XPA block ( $\mathrm{rs} 1800975$ and rs10817938; $\mathrm{D}^{\prime}=1.00, R^{2}=0.24$ ) were in LD with each other (Figure S1). Of the haplotype constructed from these blocks, only XPA GT haplotype $(\mathrm{HR}=0.68,95 \% \mathrm{CI}=0.47-0.99 ; p=0.042)$ showed statistically significant association with OS (Table S3).

Table 3. Multivariate association between nucleotide excision repair (NER) candidate single nucleotide polymorphisms (SNPs) and OSCC survival in patients treated with CCRT.

\begin{tabular}{|c|c|c|c|c|}
\hline \multirow{2}{*}{ SNPs } & \multicolumn{2}{|c|}{ Overall Survival } & \multicolumn{2}{|c|}{ Disease-Free Survival } \\
\hline & $\operatorname{HR}(95 \% \mathrm{CI})^{\mathrm{a}}$ & $p$ Value & $\operatorname{HR}(95 \% \mathrm{CI})^{b}$ & $p$ Value \\
\hline \multicolumn{5}{|l|}{$E R C C 5 / X P G$} \\
\hline \multicolumn{5}{|l|}{ rs2094258 } \\
\hline GG & 1.00 & & 1.00 & \\
\hline GA & $1.14(0.72-1.79)$ & 0.574 & $1.07(0.73-1.57)$ & 0.726 \\
\hline AA & $0.66(0.30-1.44)$ & 0.294 & $1.02(0.57-1.83)$ & 0.946 \\
\hline Additive model & $0.91(0.66-1.25)$ & 0.559 & $1.03(0.79-1.34)$ & 0.839 \\
\hline Dominant model & $1.03(0.66-1.59)$ & 0.910 & $1.06(0.74-1.52)$ & 0.754 \\
\hline Recessive model & $0.61(0.29-1.28)$ & 0.193 & $0.99(0.57-1.70)$ & 0.958 \\
\hline \multicolumn{5}{|l|}{ rs1047768 } \\
\hline TT & 1.00 & & 1.00 & \\
\hline $\mathrm{TC}$ & $1.11(0.72-1.72)$ & 0.635 & $0.93(0.64-1.36)$ & 0.715 \\
\hline $\mathrm{CC}$ & $0.63(0.25-1.62)$ & 0.339 & $0.76(0.39-1.51)$ & 0.438 \\
\hline Additive model & $0.94(0.67-1.31)$ & 0.699 & $0.90(0.68-1.19)$ & 0.453 \\
\hline Dominant model & $1.03(0.67-1.58)$ & 0.891 & $0.90(0.63-1.29)$ & 0.574 \\
\hline Recessive model & $0.60(0.24-1.49)$ & 0.272 & $0.79(0.41-1.53)$ & 0.482 \\
\hline \multicolumn{5}{|l|}{ rs17655 } \\
\hline GG & 1.00 & & 1.00 & \\
\hline GC & $0.95(0.58-1.54)$ & 0.829 & $0.86(0.56-1.31)$ & 0.482 \\
\hline $\mathrm{CC}$ & $0.93(0.51-1.69)$ & 0.811 & $1.38(0.86-2.19)$ & 0.180 \\
\hline Additive model & $0.96(0.72-1.30)$ & 0.803 & $1.16(0.91-1.49)$ & 0.238 \\
\hline Dominant model & $0.94(0.60-1.49)$ & 0.799 & $1.01(0.69-1.49)$ & 0.954 \\
\hline Recessive model & $0.96(0.58-1.61)$ & 0.881 & $1.50(1.01-2.24)$ & $0.045^{*}$ \\
\hline \multicolumn{5}{|l|}{ rs873601 } \\
\hline AA & 1.00 & & 1.00 & \\
\hline AG & $0.77(0.47-1.26)$ & 0.298 & $0.84(0.55-1.31)$ & 0.448 \\
\hline GG & $0.84(0.47-1.51)$ & 0.556 & $1.29(0.80-2.09)$ & 0.301 \\
\hline Additive model & $0.91(0.67-1.23)$ & 0.530 & $1.14(0.88-1.48)$ & 0.311 \\
\hline Dominant model & $0.79(0.50-1.26)$ & 0.304 & $0.97(0.65-1.46)$ & 0.900 \\
\hline Recessive model & $1.00(0.61-1.64)$ & 0.988 & $1.44(0.97-2.14)$ & 0.070 \\
\hline \multicolumn{5}{|l|}{$E R C C 2 / X P D$} \\
\hline \multicolumn{5}{|l|}{ rs13181 } \\
\hline $\mathrm{TT}$ & 1.00 & & 1.00 & \\
\hline TG & $1.03(0.59-1.81)$ & 0.909 & $1.00(0.61-1.64)$ & 0.997 \\
\hline GG & - & 0.989 & - & 0.984 \\
\hline Additive model & $1.03(0.59-1.80)$ & 0.921 & $1.00(0.61-1.63)$ & 0.993 \\
\hline Dominant model & $1.03(0.59-1.81)$ & 0.915 & $1.00(0.61-1.64)$ & 0.998 \\
\hline Recessive model & - & 0.989 & - & 0.984 \\
\hline \multicolumn{5}{|l|}{ rs1799793 } \\
\hline GG & 1.00 & & 1.00 & \\
\hline GA & $1.10(0.58-2.07)$ & 0.769 & $1.01(0.58-1.73)$ & 0.983 \\
\hline AA & - & 0.99 & - & - \\
\hline Additive model & $1.10(0.58-2.06)$ & 0.778 & $1.01(0.58-1.73)$ & 0.983 \\
\hline Dominant model & $1.10(0.58-2.07)$ & 0.773 & $1.01(0.58-1.73)$ & 0.983 \\
\hline Recessive model & - & 0.99 & - & - \\
\hline
\end{tabular}


Table 3. Cont.

\begin{tabular}{|c|c|c|c|c|}
\hline \multirow{2}{*}{ SNPs } & \multicolumn{2}{|c|}{ Overall Survival } & \multicolumn{2}{|c|}{ Disease-Free Survival } \\
\hline & HR $(95 \% \text { CI })^{a}$ & $p$ Value & HR $\left(95 \%\right.$ CI) ${ }^{b}$ & $p$ Value \\
\hline \multicolumn{5}{|l|}{ ERCC1 } \\
\hline \multicolumn{5}{|l|}{ rs735482 } \\
\hline AA & 1.00 & & 1.00 & \\
\hline AC & $0.72(0.46-1.14)$ & 0.163 & $0.86(0.57-1.29)$ & 0.466 \\
\hline $\mathrm{CC}$ & $0.83(0.44-1.59)$ & 0.580 & $1.47(0.89-2.44)$ & 0.134 \\
\hline Additive model & $0.86(0.62-1.19)$ & 0.352 & $1.16(0.89-1.52)$ & 0.283 \\
\hline Dominant model & $0.75(0.48-1.15)$ & 0.183 & $0.98(0.67-1.44)$ & 0.934 \\
\hline Recessive model & $1.01(0.56-1.83)$ & 0.975 & $1.61(1.04-2.51)$ & $0.034 *$ \\
\hline \multicolumn{5}{|l|}{ rs3212986 } \\
\hline GG & 1.00 & & 1.00 & \\
\hline GT & $1.20(0.76-1.89)$ & 0.436 & $0.95(0.66-1.39)$ & 0.806 \\
\hline TT & $1.04(0.48-2.28)$ & 0.922 & $0.96(0.50-1.85)$ & 0.912 \\
\hline Additive model & $1.08(0.78-1.51)$ & 0.642 & $0.97(0.73-1.29)$ & 0.833 \\
\hline Recessive model & $1.17(0.75-1.82)$ & 0.481 & $0.96(0.67-1.37)$ & 0.805 \\
\hline Dominant model & $0.94(0.45-1.97)$ & 0.870 & $0.99(0.53-1.85)$ & 0.967 \\
\hline \multicolumn{5}{|l|}{ rs11615 } \\
\hline $\mathrm{CC}$ & 1.00 & & 1.00 & \\
\hline $\mathrm{CT}$ & $1.23(0.79-1.91)$ & 0.351 & $0.85(0.59-1.23)$ & 0.388 \\
\hline TT & $0.90(0.35-2.28)$ & 0.817 & $0.72(0.33-1.59)$ & 0.416 \\
\hline Additive model & $1.08(0.77-1.51)$ & 0.661 & $0.85(0.63-1.14)$ & 0.282 \\
\hline Dominant model & $1.18(0.77-1.81)$ & 0.438 & $0.83(0.58-1.19)$ & 0.316 \\
\hline Recessive model & $0.81(0.33-2.01)$ & 0.652 & $0.78(0.36-1.69)$ & 0.525 \\
\hline \multicolumn{5}{|l|}{$X P C$} \\
\hline \multicolumn{5}{|l|}{ rs2228001 } \\
\hline AA & 1.00 & & 1.00 & \\
\hline $\mathrm{AC}$ & $1.07(0.68-1.69)$ & 0.766 & $1.21(0.83-1.78)$ & 0.329 \\
\hline $\mathrm{CC}$ & $0.72(0.30-1.73)$ & 0.457 & $0.75(0.37-1.54)$ & 0.432 \\
\hline Additive model & $0.94(0.67-1.32)$ & 0.716 & $0.99(0.75-1.31)$ & 0.941 \\
\hline Dominant model & $1.01(0.65-1.58)$ & 0.955 & $1.12(0.77-1.63)$ & 0.539 \\
\hline Recessive model & $0.69(0.30-1.60)$ & 0.384 & $0.68(0.34-1.34)$ & 0.260 \\
\hline \multicolumn{5}{|l|}{ rs2228000 } \\
\hline $\mathrm{CC}$ & 1.00 & & 1.00 & \\
\hline TC & $1.06(0.66-1.68)$ & 0.822 & $0.81(0.55-1.18)$ & 0.267 \\
\hline $\mathrm{TT}$ & $1.86(0.97-3.56)$ & 0.062 & $1.11(0.59-2.08)$ & 0.758 \\
\hline Additive model & $1.28(0.92-1.77)$ & 0.144 & $0.94(0.70-1.25)$ & 0.652 \\
\hline Dominant model & $1.18(0.76-1.83)$ & 0.457 & $0.85(0.59-1.22)$ & 0.373 \\
\hline Recessive model & $1.81(0.99-3.29)$ & 0.053 & $1.23(0.67-2.26)$ & 0.501 \\
\hline \multicolumn{5}{|l|}{$X P A$} \\
\hline \multicolumn{5}{|l|}{ rs1800975 } \\
\hline AA & 1.00 & & 1.00 & \\
\hline AG & $0.83(0.50-1.36)$ & 0.461 & $0.83(0.51-1.36)$ & 0.462 \\
\hline GG & $0.65(0.35-1.21)$ & 0.175 & $0.65(0.35-1.21)$ & 0.174 \\
\hline Additive model & $0.81(0.60-1.10)$ & 0.174 & $0.90(0.70-1.16)$ & 0.426 \\
\hline Dominant model & $0.77(0.48-1.24)$ & 0.282 & $0.77(0.48-1.24)$ & 0.283 \\
\hline Recessive model & $0.74(0.44-1.25)$ & 0.260 & $0.73(0.44-1.25)$ & 0.258 \\
\hline \multicolumn{5}{|l|}{ rs10817938 } \\
\hline TT & 1.00 & & 1.00 & \\
\hline TC & $1.08(0.68-1.72)$ & 0.731 & $1.28(0.87-1.86)$ & 0.209 \\
\hline $\mathrm{CC}$ & $1.68(0.65-4.33)$ & 0.286 & $1.88(0.75-4.74)$ & 0.180 \\
\hline Additive model & $1.18(0.82-1.69)$ & 0.386 & $1.31(0.95-1.80)$ & 0.095 \\
\hline Dominant model & $1.15(0.74-1.77)$ & 0.543 & $1.32(0.91-1.90)$ & 0.139 \\
\hline Recessive model & $1.64(0.64-4.17)$ & 0.304 & $1.73(0.69-4.32)$ & 0.239 \\
\hline
\end{tabular}

OSCC, oral squamous cell carcinoma; SNPs, single nucleotide polymorphisms; HR, hazard ratio; CI, confidence interval. a Adjusted for age, BMI, N stage, lymphatic invasion, and extranodal extension. ${ }^{\mathrm{b}}$ Adjusted for age, $\mathrm{T}$ stage, $\mathrm{N}$ stage, and extranodal extension. ${ }^{*} p<0.05$.

We further conducted a combination analysis for the ERCC5 rs17655 and ERCC1 rs735482 polymorphisms and DSF in patients with OSCC. The Kaplan-Meier curves showed borderline significant differences in DFS among the four genotypes (log-rank test $p=0.078$ ) (Figure 1). The multivariate Cox proportional models indicated that patients with the combination of ERCC5 rs17655 CC and ERCC1 rs735482 CC genotypes exhibited a higher risk of disease recurrence than those with the combination of ERCC5 rs17655 GG+GC and ERCC1 rs735482 AA+AC genotypes $(\mathrm{HR}=2.60,95 \% \mathrm{CI}=1.11-6.09 ; p=0.027)$ (Table 4). However, this gene-gene interaction was not statistically significant. 


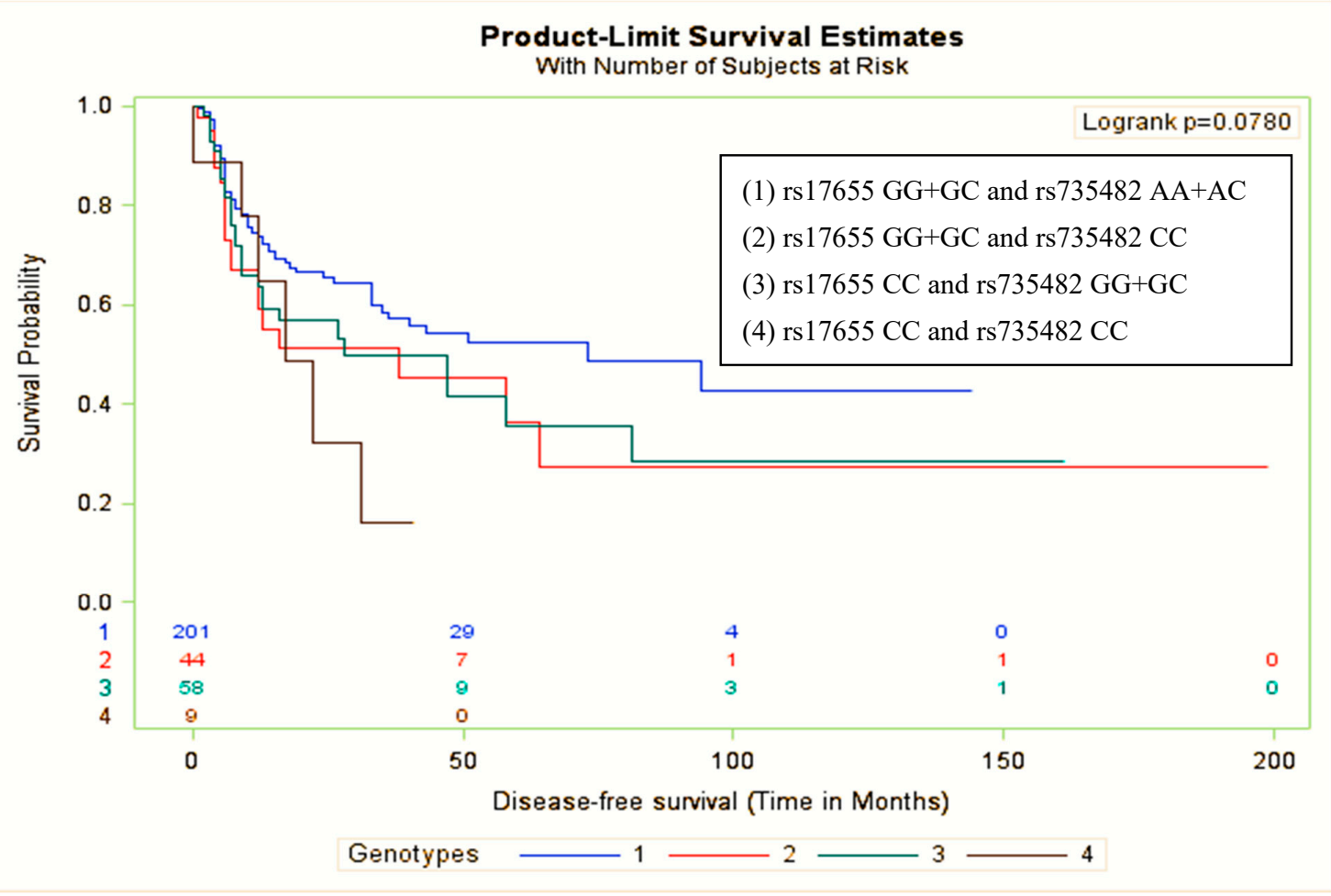

Figure 1. Kaplan-Meier survival curve for the combined ERCC5 rs17655 and ERCC1 rs735482 polymorphisms and disease-free survival in patients with oral squamous cell carcinoma treated with concurrent chemoradiotherapy. The figure illustrates a borderline significant difference in recurrence among the four groups (log-rank test $p=0.078$ ).

Table 4. Interaction between the ERCC5 rs17655 and ERCC1 rs735482 polymorphisms on the disease-free survival of patients with OSCC treated with CCRT.

\begin{tabular}{cccccc}
\hline ERCC5 rs17655 & ERCC1 rs735482 & No. & Event & HR (95\% CI) ${ }^{\text {a }}$ & $p$ Value \\
\hline GG+GC & AA+AC & 206 & 71 & 1.00 & \\
GG+GC & CC & 45 & 19 & $1.63(0.98-2.72)$ & 0.060 \\
CC & GG+GC & 59 & 29 & $1.52(0.98-2.37)$ & 0.062 \\
CC & CC & 9 & 6 & $2.60(1.11-6.09)$ & $0.027^{*}$ \\
& $p$ for interaction & & & 0.929 &
\end{tabular}

OSCC, oral squamous cell carcinoma; HR, hazard ratio; CI, confidence interval. a Adjusted for age, $\mathrm{T}$ stage, $\mathrm{N}$ stage, and extranodal extension. ${ }^{*} p<0.05$.

Table 5 shows the subgroup analysis for the association between significant SNPs and DFS stratified by demographic and clinopathological factors. Results show a significant interaction between ERCC5 rs17655 polymorphism and perineural invasion on the risk for DFS (interaction $p=0.008$ ). The ERCC5 rs17655 CC genotype individuals with perineural invasion $(\mathrm{HR}=2.46,95 \% \mathrm{CI}=1.46-4.15$; $p<0.001)$ had an increased risk for DFS compared to their counterparts with no perineural invasion. Although a significant interaction between ERCC1 rs735482 polymorphism and vascular invasion was also observed (interaction $p<0.001$ ), the harmful effect of CC genotype on recurrence was not present in any subgroup of vascular invasion. 
Table 5. Association between the ERCC5 rs17655 and ERCC1 rs735482 polymorphisms and the disease-free survival in OSCC patients treated with CCRT stratified by demographic and clinopathological factors.

\begin{tabular}{|c|c|c|c|c|c|c|}
\hline \multirow[b]{2}{*}{ Variable } & \multicolumn{2}{|c|}{ ERCC5 rs17655 } & \multicolumn{4}{|c|}{ ERCC1 rs735482 } \\
\hline & $\operatorname{HR}(95 \% \mathrm{CI})^{\mathrm{a}}$ & $p$ Value & $p_{\text {Interaction }}$ & $\operatorname{HR}(95 \% \mathrm{CI})^{\mathrm{a}}$ & $p$ Value & $p_{\text {Interaction }}$ \\
\hline Age & & & 0.486 & & & 0.078 \\
\hline$<50$ & $1.74(1.00-3.03)$ & 0.051 & & $1.04(0.53-2.06)$ & 0.911 & \\
\hline$\geq 50$ & $1.24(0.69-2.23)$ & 0.473 & & $2.76(1.47-5.19)$ & $0.002 *$ & \\
\hline BMI, $\mathrm{kg} / \mathrm{m}^{2}$ & & & 0.160 & & & 0.597 \\
\hline $18.5-23.9$ & $1.64(0.95-2.82)$ & 0.075 & & $1.89(1.04-3.44)$ & $0.037 *$ & \\
\hline$<18.5$ & $3.06(0.65-14.49)$ & 0.159 & & $1.30(0.05-31.65)$ & 0.871 & \\
\hline$\geq 24$ & $0.81(0.37-1.77)$ & 0.601 & & $1.30(0.62-2.72)$ & 0.495 & \\
\hline Cigarette smoking & & & 0.664 & & & 0.975 \\
\hline Never & $1.03(0.35-2.98)$ & 0.962 & & $1.99(0.52-7.58)$ & 0.316 & \\
\hline Ever & $1.54(0.99-2.41)$ & 0.056 & & $1.61(1.00-2.59)$ & 0.051 & \\
\hline Alcohol drinking & & & 0.993 & & & 0.676 \\
\hline Never & $1.59(0.74-3.42)$ & 0.240 & & $1.43(0.64-3.22)$ & 0.387 & \\
\hline Ever & $1.52(0.94-2.46)$ & 0.091 & & $1.72(1.00-2.96)$ & 0.050 & \\
\hline Betel nut chewing & & & 0.445 & & & 0.968 \\
\hline Never & $2.06(0.53-8.11)$ & 0.299 & & $1.64(0.34-7.86)$ & 0.536 & \\
\hline Ever & $1.41(0.92-2.14)$ & 0.112 & & $1.63(1.02-2.62)$ & $0.043 *$ & \\
\hline Tea drinking & & & 0.331 & & & 0.986 \\
\hline Never & $1.88(1.05-3.35)$ & $0.033 *$ & & $1.60(0.87-2.94)$ & 0.134 & \\
\hline Ever & $1.27(0.73-2.24)$ & 0.400 & & $1.57(0.81-3.04)$ & 0.183 & \\
\hline Coffee drinking & & & 0.073 & & & 0.077 \\
\hline Never & $1.30(0.82-2.08)$ & 0.066 & & $2.09(1.28-3.41)$ & $0.003 *$ & \\
\hline Ever & $2.83(1.27-6.33)$ & $0.011 *$ & & $0.72(0.22-2.42)$ & 0.601 & \\
\hline Tumor differentiation & & & 0.492 & & & 0.949 \\
\hline Well differentiated & $1.08(0.43-2.68)$ & 0.875 & & $3.74(1.26-11.09)$ & $0.018^{*}$ & \\
\hline Moderate & $1.43(0.84-2.42)$ & 0.184 & & $1.06(0.57-1.98)$ & 0.858 & \\
\hline Poor & $2.15(0.88-5.22)$ & 0.093 & & $3.48(1.23-9.88)$ & $0.019 *$ & \\
\hline Primary tumor size & & & 0.146 & & & 0.394 \\
\hline $\mathrm{T} 1-\mathrm{T} 2$ & $0.96(0.45-2.03)$ & 0.905 & & $1.12(0.49-2.56)$ & 0.788 & \\
\hline T3-T4 & $1.83(1.14-2.93)$ & $0.013 *$ & & $1.76(1.04-3.00)$ & $0.036^{*}$ & \\
\hline Nodal involvement & & & 0.328 & & & 0.125 \\
\hline N0-N1 & $2.25(1.06-4.81)$ & $0.036 *$ & & $3.16(1.47-6.80)$ & $0.003 *$ & \\
\hline N2-N3 & $1.32(0.82-2.13)$ & 0.252 & & $1.23(0.69-2.19)$ & 0.479 & \\
\hline Perineural invasion & & & $0.008 *$ & & & 0.416 \\
\hline No & $0.77(0.40-1.49)$ & 0.429 & & $2.07(1.06-4.03)$ & $0.032 *$ & \\
\hline Yes & $2.46(1.46-4.15)$ & $<0.001 *$ & & $1.32(0.72-2.44)$ & 0.370 & \\
\hline Vascular invasion & & & 0.410 & & & $<0.001 *$ \\
\hline No & $1.45(0.96-2.19)$ & 0.078 & & $1.51(0.96-2.37)$ & 0.076 & \\
\hline Yes & $1.27(0.16-10.34)$ & 0.826 & & - & & \\
\hline Lymphatic invasion & & & 0.553 & & & 0.449 \\
\hline No & $1.43(0.92-2.23)$ & 0.111 & & $1.71(1.07-2.73)$ & $0.025 *$ & \\
\hline Yes & $1.84(0.64-5.31)$ & 0.260 & & $0.90(0.20-4.16)$ & 0.892 & \\
\hline Extranodal extension & & & 0.253 & & & 0.720 \\
\hline No & $2.35(1.12-4.94)$ & $0.024 *$ & & $2.17(0.87-5.40)$ & 0.097 & \\
\hline Yes & $1.31(0.81-2.13)$ & 0.277 & & $1.52(0.90-2.57)$ & 0.118 & \\
\hline Pathologic TNM stage & & & 0.850 & & & 0.585 \\
\hline III & $1.70(0.48-6.09)$ & 0.414 & & $4.82(0.98-23.58)$ & 0.053 & \\
\hline IV & $1.51(0.99-2.31)$ & 0.055 & & $1.54(0.96-2.47)$ & 0.075 & \\
\hline
\end{tabular}

OSCC, oral squamous cell carcinoma; HR, hazard ratio; CI, confidence interval; Int, interaction. ${ }^{a}$ Adjusted for age, $\mathrm{T}$ stage, $\mathrm{N}$ stage, and extranodal extension. ${ }^{*} p<0.05$.

\section{Discussion}

In this study, we investigated the association between potentially functional SNPs in the NER pathway genes and clinical outcomes in male patients with OSCC treated with CCRT. Our findings suggest that the XPC rs2228000 TT genotype was marginally significantly associated with increased risk of death, whereas the ERCC5 rs17655 CC and ERCC1 rs735482 CC genotypes were significantly associated with the increased risk of relapse.

The NER pathway plays a major role in DNA repair through the removal of bulky DNA lesions formed by ultraviolet (UV) radiation, environmental mutagens, and other chemotherapeutic agents $[23,24]$. Studies have revealed that variations in DNA-repair capacity are related to cancer risk and prognosis $[25,26]$. In addition, SNPs in the NER genes modulate susceptibility to efficacy and survival outcome of the treatment in certain types of cancers [11,12]. Therefore, the same phenomena 
may be exhibited in patients with OSCC, particularly those undergoing CCRT. Such information may be useful in identifying patients who may benefit from alternative therapies to achieve superior survival and improve quality of life.

The XPC gene is a key component of the XPC complex, which plays an important role in the early part of the global genome NER. The corresponding protein plays an important function in damage sensing and DNA binding [27]. SNPs in this gene have been found to affect clinical outcomes in various cancer types $[18,28,29]$. Li and colleagues observed that the XPC rs2228000 TT genotypes were associated with shorter OS than the CC+CT genotype individuals in a study of Japanese gastric cancer patients [30]. Another Chinese study demonstrated that patients with the CC genotype of $X P C$ rs2228000 have a borderline significant decreased risk of developing gastric cancer compared with those with the CT+TT genotype [31]. This evidence suggests that the T-allele may have a high susceptibility for poor prognosis. Similarly, in our study, the XPC rs2228000 TT genotype shows an increased risk of death compared with the $C C+C T$ genotype. Given the importance of the XPC gene in the NER pathway, it is possible that variants of XPC alter the DNA repair capacity and thereby affect sensitivity to therapeutic agents. However, the association observed in our study was borderline significant and must be interpreted with caution. Furthermore, large studies may be required to confirm these findings.

We also observed that those with the ERCC5 rs17655 CC and ERCC1 rs735482 CC genotypes have an increased risk of relapse compared with individuals with the GG+GC and AA+AC genotypes, respectively. Considered a central component in NER, ERCC5 encodes a specific DNA endonuclease responsible for excision and repair of UV-induced DNA damage [23]. Evidence has linked ERCC5 polymorphism to chemotherapeutic response and prognosis of tumors $[19,20]$. The ERCC5 mRNA expression levels were correlated with cytotoxicity of chemotherapy regiments [32]. Additionally, the rs17655 leads to an amino-acid substitution from histidine to aspartic acid, which may lead to differential interacting abilities, thus, influencing the DNA repair efficacy. Song et al. also observed that ERCC5 rs17655 polymorphism has a moderately increased risk of recurrence in squamous cell carcinoma of the oropharynx [33]. ERCC1 is also a crucial member of the NER pathway that forms a complex with ERCC4, and together with ERCC5, is responsible for DNA incision [34]. Other studies have reported that ERCC1 affects the clinical outcome and may serve as a potential biomarker for response to cisplatin-based therapy [35,36]. On the basis of these study results, we speculate that the CC genotypes of ERCC5 rs17655 and ERCC1 rs735482 may increase the DNA-repair capacity of cancer cells, leading to increased susceptibility to recurrence. Therefore, if these findings are confirmed by other studies, these SNPs may serve as therapeutic biomarkers for clinical outcome in patients with OSCC who undergo CCRT.

Our study has several limitations. First, the hospital-based nature of the patients may have led to selection bias. Secondly, not all SNPs in the entire NER pathway were used. Some rare functional SNPs may have an influence on survival. Finally, the human papilloma virus (HPV) status and inflammatory cytokines expression of patients was not included in the analysis and may limit the interpretation of our findings; hence, HPV and cytokines may affect survival [37,38]. However, a major strength of our study is that all the patients had a similar tumor stage and received the same treatment. This meant that the effect of different treatments was excluded, which might lead to different levels of DNA damage and repair.

\section{Conclusions}

We investigated the association between key potentially functional SNPs in the NER pathway and susceptibility for death or relapse in male patients with advanced OSCC who were treated with adjuvant CCRT. Our findings showed that the CC genotypes of ERCC5 rs17655 and ERCC1 rs735482 were associated with an increased risk of recurrence. CCRT may not be beneficial for these patients; therefore, alternative treatments are required. To our knowledge, this is the largest study to investigate the association between NER polymorphisms and survival in patients with OSCC treated with CCRT 
in ethnic Chinese. Our findings may require further confirmation in studies with a larger sample size or other ethnic populations.

Supplementary Materials: The following are available online at http:/ /www.mdpi.com/2077-0383/8/1/33/s1, Figure S1: Linkage disequilibrium (LD) analysis among single nucleotide polymorphisms (SNPs) within the same chromosome or gene, Table S1: Frequency distributions of NER polymorphisms by clinicopathological factors, Table S2: Univariate association between NER candidate SNPs and OSCC survival in CCRT treated patients, Table S3: Haplotype analysis of association between NER candidate SNPs and OSCC survival in patients treated with CCRT.

Author Contributions: Conceptualization: S.-F.H. and C.-C.Y.; investigation: H.-T.C. and C.-K.Y.; methodology: T.S., Y.-X.C., W.-C.W., S.-F.H., and C.-C.Y.; resources: H.-T.C., Y.-X.C., and C.-K.Y.; formal analysis: T.S. and Y.-X.C.; validation: T.S., H.-T.C., and Y.-X.C.; writing: (original draft preparation) T.S.; writing (review and editing): T.S., W.-C.W., S.-F.H., and C.-C.Y.; supervision: S.-F.H. and C.-C.Y.; and funding acquisition: S.-F.H. and C.-C.Y.

Funding: The research was supported by the Ministry of Science and Technology, Taiwan (MOST 107-2314-B-038-071).

Acknowledgments: The authors would like to thank Wallace Academic Editing for the English language review.

Conflicts of Interest: The authors declare no conflict of interest.

\section{References}

1. Chiang, C.-J.; Lo, W.-C.; Yang, Y.-W.; You, S.-L.; Chen, C.-J.; Lai, M.-S. Incidence and survival of adult cancer patients in Taiwan, 2002-2012. J. Formos. Med. Assoc. 2016, 115, 1076-1088. [CrossRef] [PubMed]

2. Amit, M.; Yen, T.C.; Liao, C.T.; Chaturvedi, P.; Agarwal, J.P.; Kowalski, L.P.; Ebrahimi, A.; Clark, J.R.; Kreppel, M.; Zoller, J.; et al. Improvement in survival of patients with oral cavity squamous cell carcinoma: An international collaborative study. Cancer 2013, 119, 4242-4248. [CrossRef] [PubMed]

3. Leemans, C.R.; Tiwari, R.; Nauta, J.J.; van der Waal, I.; Snow, G.B. Recurrence at the primary site in head and neck cancer and the significance of neck lymph node metastases as a prognostic factor. Cancer 1994, 73, 187-190. [CrossRef]

4. Markopoulos, A.K. Current Aspects on Oral Squamous Cell Carcinoma. Open Dent. J. 2012, 6, 126-130. [CrossRef] [PubMed]

5. $\quad$ Adelstein, D.; Gillison, M.L.; Pfister, D.G.; Spencer, S.; Adkins, D.; Brizel, D.M.; Burtness, B.; Busse, P.M.; Caudell, J.J.; Cmelak, A.J.; et al. NCCN Guidelines Insights: Head and Neck Cancers, Version 2.2017. J. Natl. Compr. Cancer Netw. 2017, 15, 761-770. [CrossRef] [PubMed]

6. Otsuru, M.; Ota, Y.; Aoki, T.; Sasaki, M.; Suzuki, T.; Denda, Y.; Takahashi, M.; Akiba, T.; Kaneko, A. A Study of Adjuvant Chemoradiotherapy with Tri-weekly Cisplatin for Postoperative High-risk Oral Squamous Cell Carcinoma. Tokai J. Exp. Clin. Med. 2017, 42, 19-24. [PubMed]

7. Ferris, R.L.; Geiger, J.L.; Trivedi, S.; Schmitt, N.C.; Heron, D.E.; Johnson, J.T.; Kim, S.; Duvvuri, U.; Clump, D.A.; Bauman, J.E.; et al. Phase II trial of post-operative radiotherapy with concurrent cisplatin plus panitumumab in patients with high-risk, resected head and neck cancer. Ann. Oncol. 2016, 27, 2257-2262. [CrossRef]

8. Bernier, J.; Domenge, C.; Ozsahin, M.; Matuszewska, K.; Lefebvre, J.L.; Greiner, R.H.; Giralt, J.; Maingon, P.; Rolland, F.; Bolla, M.; et al. Postoperative irradiation with or without concomitant chemotherapy for locally advanced head and neck cancer. N. Engl. J. Med. 2004, 350, 1945-1952. [CrossRef]

9. Tanvetyanon, T.; Padhya, T.; McCaffrey, J.; Kish, J.A.; Deconti, R.C.; Trotti, A.; Rao, N.G. Postoperative concurrent chemotherapy and radiotherapy for high-risk cutaneous squamous cell carcinoma of the head and neck. Head Neck 2015, 37, 840-845. [CrossRef]

10. Leeman, J.E.; Li, J.G.; Pei, X.; Venigalla, P.; Zumsteg, Z.S.; Katsoulakis, E.; Lupovitch, E.; McBride, S.M.; Tsai, C.J.; Boyle, J.O.; et al. Patterns of Treatment Failure and Postrecurrence Outcomes Among Patients With Locally Advanced Head and Neck Squamous Cell Carcinoma After Chemoradiotherapy Using Modern Radiation Techniques. JAMA Oncol. 2017, 3, 1487-1494. [CrossRef]

11. Jin, H.; Xie, X.; Wang, H.; Hu, J.; Liu, F.; Liu, Z.; Zhou, J.; Zhang, Y.; Xi, X.; Hu, B.; et al. ERCC1 Cys8092Ala and XRCC1 Arg399Gln polymorphisms predict progression-free survival after curative radiotherapy for nasopharyngeal carcinoma. PLoS ONE 2014, 9, e101256. [CrossRef] [PubMed] 
12. Liu, H.; Qi, B.; Guo, X.; Tang, L.Q.; Chen, Q.Y.; Zhang, L.; Guo, L.; Luo, D.H.; Huang, P.Y.; Mo, H.Y.; et al. Genetic variations in radiation and chemotherapy drug action pathways and survival in locoregionally advanced nasopharyngeal carcinoma treated with chemoradiotherapy. PLoS ONE 2013, 8, e82750. [CrossRef] [PubMed]

13. Lopes-Aguiar, L.; Costa, E.F.D.; Nogueira, G.A.S.; Lima, T.R.P.; Visacri, M.B.; Pincinato, E.C.; Calonga, L.; Mariano, F.V.; de Almeida Milani Altemani, A.M.; Altemani, J.M.C.; et al. XPD c.934G>A polymorphism of nucleotide excision repair pathway in outcome of head and neck squamous cell carcinoma patients treated with cisplatin chemoradiation. Oncotarget 2016, 8, 16190-16201. [CrossRef] [PubMed]

14. Nanda, S.S.; Gandhi, A.K.; Rastogi, M.; Khurana, R.; Hadi, R.; Sahni, K.; Mishra, S.P.; Srivastava, A.K.; Bhatt, M.L.B.; Parmar, D. Evaluation of XRCC1 Gene Polymorphism as a Biomarker in Head and Neck Cancer Patients Undergoing Chemoradiation Therapy. Int. J. Radiat. Oncol. Biol. Phys. 2018, 101, 593-601. [CrossRef]

15. Rouillon, C.; White, M.F. The evolution and mechanisms of nucleotide excision repair proteins. Res. Microbiol. 2011, 162, 19-26. [CrossRef] [PubMed]

16. Gao, C.; Wang, J.; Li, C.; Zhang, W.; Liu, G. A Functional Polymorphism (rs10817938) in the XPA Promoter Region Is Associated with Poor Prognosis of Oral Squamous Cell Carcinoma in a Chinese Han Population. PLoS ONE 2016, 11, e0160801. [CrossRef] [PubMed]

17. Tan, L.M.; Qiu, C.F.; Zhu, T.; Jin, Y.X.; Li, X.; Yin, J.Y.; Zhang, W.; Zhou, H.H.; Liu, Z.Q. Genetic Polymorphisms and Platinum-based Chemotherapy Treatment Outcomes in Patients with Non-Small Cell Lung Cancer: A Genetic Epidemiology Study Based Meta-analysis. Sci. Rep. 2017, 7, 5593. [CrossRef]

18. Wang, C.; Nie, H.; Li, Y.; Liu, G.; Wang, X.; Xing, S.; Zhang, L.; Chen, X.; Chen, Y.; Li, Y. The study of the relation of DNA repair pathway genes SNPs and the sensitivity to radiotherapy and chemotherapy of NSCLC. Sci. Rep. 2016, 6, 26526. [CrossRef]

19. Zhang, R.; Zhou, F.; Cheng, L.; Yu, A.; Zhu, M.; Wang, M.; Zhang, Z.; Xiang, J.; Wei, Q. Genetic variants in nucleotide excision repair pathway predict survival of esophageal squamous cell cancer patients receiving platinum-based chemotherapy. Mol. Carcinog. 2018, 57, 1553-1565. [CrossRef]

20. Zhou, F.; Zhu, M.; Wang, M.; Qiu, L.; Cheng, L.; Jia, M.; Xiang, J.; Wei, Q. Genetic variants of DNA repair genes predict the survival of patients with esophageal squamous cell cancer receiving platinum-based adjuvant chemotherapy. J. Transl. Med. 2016, 14, 154. [CrossRef]

21. Amin, M.B.; Greene, F.L.; Edge, S.B.; Compton, C.C.; Gershenwald, J.E.; Brookland, R.K.; Meyer, L.; Gress, D.M.; Byrd, D.R.; Winchester, D.P. The Eighth Edition AJCC Cancer Staging Manual: Continuing to build a bridge from a population-based to a more "personalized" approach to cancer staging. CA: A Cancer J. Clin. 2017, 67, 93-99. [CrossRef] [PubMed]

22. Stephens, M.; Smith, N.J.; Donnelly, P. A new statistical method for haplotype reconstruction from population data. Am. J. Hum. Genet. 2001, 68, 978-989. [CrossRef] [PubMed]

23. Schärer, O.D. Nucleotide Excision Repair in Eukaryotes. Cold Spring Harb. Perspect. Biol. 2013, 5, a012609. [CrossRef] [PubMed]

24. Friedberg, E.C. How nucleotide excision repair protects against cancer. Nat. Rev. Cancer 2001, 1, $22-33$. [CrossRef] [PubMed]

25. Naccarati, A.; Rosa, F.; Vymetalkova, V.; Barone, E.; Jiraskova, K.; Di Gaetano, C.; Novotny, J.; Levy, M.; Vodickova, L.; Gemignani, F.; et al. Double-strand break repair and colorectal cancer: Gene variants within $3^{\prime}$ UTRs and microRNAs binding as modulators of cancer risk and clinical outcome. Oncotarget 2016, 7, 23156-23169. [CrossRef]

26. Mucha, B.; Pytel, D.; Markiewicz, L.; Cuchra, M.; Szymczak, I.; Przybylowska-Sygut, K.; Dziki, A.; Majsterek, I.; Dziki, L. Nucleotide Excision Repair Capacity and XPC and XPD Gene Polymorphism Modulate Colorectal Cancer Risk. Clin. Colorectal. Cancer 2018, 17, e435-e441. [CrossRef] [PubMed]

27. Sugasawa, K.; Ng, J.M.; Masutani, C.; Iwai, S.; van der Spek, P.J.; Eker, A.P.; Hanaoka, F.; Bootsma, D.; Hoeijmakers, J.H. Xeroderma pigmentosum group C protein complex is the initiator of global genome nucleotide excision repair. Mol. Cell 1998, 2, 223-232. [CrossRef]

28. Zheng, Y.; Deng, Z.; Yin, J.; Wang, S.; Lu, D.; Wen, X.; Li, X.; Xiao, D.; Hu, C.; Chen, X.; et al. The association of genetic variations in DNA repair pathways with severe toxicities in NSCLC patients undergoing platinum-based chemotherapy. Int. J. Cancer 2017, 141, 2336-2347. [CrossRef] [PubMed] 
29. Ravegnini, G.; Nannini, M.; Simeon, V.; Musti, M.; Sammarini, G.; Saponara, M.; Gatto, L.; Urbini, M.; Astolfi, A.; Biasco, G.; et al. Polymorphisms in DNA repair genes in gastrointestinal stromal tumours: Susceptibility and correlation with tumour characteristics and clinical outcome. Tumour Biol. 2016, 37, 13413-13423. [CrossRef]

30. Li, Y.; Liu, Z.; Liu, H.; Wang, L.E.; Onodera, H.; Suzuki, A.; Suzuki, K.; Wadhwa, R.; Elimova, E.; Sudo, K.; et al. Potentially functional variants in the core nucleotide excision repair genes predict survival in Japanese gastric cancer patients. Carcinogenesis 2014, 35, 2031-2038. [CrossRef]

31. Hua, R.-X.; Zhuo, Z.-J.; Shen, G.-P.; Zhu, J.; Zhang, S.-D.; Xue, W.-Q.; Li, X.-Z.; Zhang, P.-F.; He, J.; Jia, W.-H. Polymorphisms in the XPC gene and gastric cancer susceptibility in a Southern Chinese population. Onco Targets Ther. 2016, 9, 5513-5519. [CrossRef] [PubMed]

32. Zhao, Y.L.; Yang, L.B.; Geng, X.L.; Zhou, Q.L.; Qin, H.; Yang, L.; Dong, Y.Z.; Zhong, J.J. The association of XPG and MMS19L polymorphisms response to chemotherapy in osteosarcoma. Pak. J. Med. Sci. 2013, 29, 1225-1229. [CrossRef] [PubMed]

33. Song, X.; Sturgis, E.M.; Jin, L.; Wang, Z.; Wei, Q.; Li, G. Variants in nucleotide excision repair core genes and susceptibility to recurrence of squamous cell carcinoma of the oropharynx. Int. J. Cancer 2013, 133, 695-704. [CrossRef] [PubMed]

34. Bowden, N.A. Nucleotide excision repair: Why is it not used to predict response to platinum-based chemotherapy? Cancer Lett. 2014, 346, 163-171. [CrossRef] [PubMed]

35. Arriagada, R.; Bergman, B.; Dunant, A.; Le Chevalier, T.; Pignon, J.P.; Vansteenkiste, J. Cisplatin-based adjuvant chemotherapy in patients with completely resected non-small-cell lung cancer. N. Engl. J. Med. 2004, 350, 351-360. [CrossRef] [PubMed]

36. Olaussen, K.A.; Dunant, A.; Fouret, P.; Brambilla, E.; Andre, F.; Haddad, V.; Taranchon, E.; Filipits, M.; Pirker, R.; Popper, H.H.; et al. DNA repair by ERCC1 in non-small-cell lung cancer and cisplatin-based adjuvant chemotherapy. N. Engl. J. Med. 2006, 355, 983-991. [CrossRef] [PubMed]

37. Lafaurie, G.I.; Perdomo, S.J.; Buenahora, M.R.; Amaya, S.; Diaz-Baez, D. Human papilloma virus: An etiological and prognostic factor for oral cancer? J. Investig. Clin. Dent. 2018, 9, e12313. [CrossRef]

38. Kwon, M.; Kim, J.W.; Roh, J.L.; Park, Y.; Cho, K.J.; Choi, S.H.; Nam, S.Y.; Kim, S.Y.; Lee, B.H. Recurrence and cancer-specific survival according to the expression of IL-4Ralpha and IL-13Ralpha1 in patients with oral cavity cancer. Eur. J. Cancer (Oxf. Engl.: 1990) 2015, 51, 177-185. [CrossRef] 